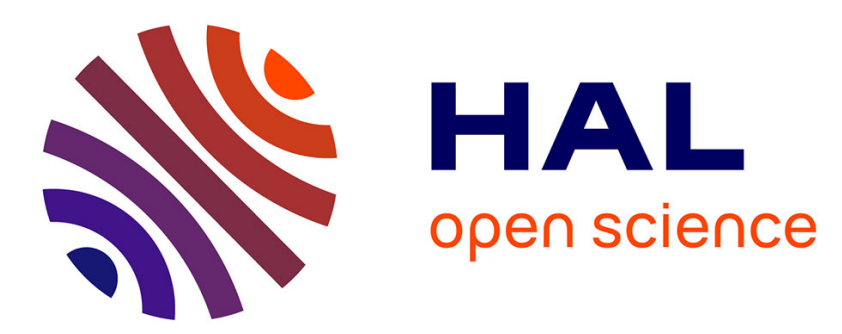

\title{
Generic 3D Geometrical and Mechanical Modeling of the Skin/Subcutaneous Complex by a Procedural Hybrid Method
}

Christian Herlin, Benjamin Gilles, Gérard Subsol, Guillaume Captier

\section{To cite this version:}

Christian Herlin, Benjamin Gilles, Gérard Subsol, Guillaume Captier. Generic 3D Geometrical and Mechanical Modeling of the Skin/Subcutaneous Complex by a Procedural Hybrid Method. ISBMS: International Symposium on Biomedical Simulation, Oct 2014, Strasbourg, France. pp.173-181, 10.1007/978-3-319-12057-7_20 . lirmm-01239192

\section{HAL Id: lirmm-01239192 \\ https://hal-lirmm.ccsd.cnrs.fr/lirmm-01239192}

Submitted on 7 Dec 2015

HAL is a multi-disciplinary open access archive for the deposit and dissemination of scientific research documents, whether they are published or not. The documents may come from teaching and research institutions in France or abroad, or from public or private research centers.
L'archive ouverte pluridisciplinaire HAL, est destinée au dépôt et à la diffusion de documents scientifiques de niveau recherche, publiés ou non, émanant des établissements d'enseignement et de recherche français ou étrangers, des laboratoires publics ou privés. 


\title{
Generic 3D Geometrical and Mechanical Modeling of the Skin/Subcutaneous Complex by a Procedural Hybrid Method
}

\author{
Christian Herlin ${ }^{1,2,3}$, Benjamin Gilles ${ }^{4}$, Gérard Subsol ${ }^{4}$, \\ and Guillaume Captier ${ }^{1,3}$ \\ 1 Dept. of Plastic Pediatric Surgery, CHRU Montpellier, France \\ c-herlin@chu-montpellier.fr \\ 2 Dept. of Plastic Surgery, Burns and Wound Healing,CHRU Montpellier, France \\ 3 Laboratory of Anatomy, Montpellier 1 University, France \\ 4 ICAR Research Team, LIRMM, CNRS/University of Montpellier 2, France
}

\begin{abstract}
The aim of this work is to build a 3D geometric and mechanical model of the skin/subcutaneous complex (SSC) which could be adapted to the different parts of the body and to the morphological parameters of the patient. We present first the anatomical pattern of the SSC. Then, we propose a hybrid model which combines volume, membranous and unidimensional models. The complex internal structure of the SSC is automatically created by a procedural process. All the models are defined by some parameters which can be easily measured by medical imaging. We describe several preliminary experiments which show how this hybrid method models realistic geometrical deformations and physical behaviors and could be used for surgery simulation and planning.
\end{abstract}

Keywords: biomechanics, soft tissue, skin/subcutaneous complex, plastic surgery, procedural method.

\section{Introduction}

Surgeons, biomechanicians and digital designers are all looking for accurate, realistic means of predicting the deformation of soft tissues. Although different objectives have been pursued in each case, the main problem arising has been that of modelling a heterogeneous three-dimensional structure showing complex mechanical behavior. In fact, there exists no mechanical model so far which accurately simulates the anatomy of the skin/subcutaneous complex (SSC), taking the connective arrangements and the mobility of the components into account.

Most models are uni- or bi-lamellar, composed of layers which often have a constant thickness and which show linear isotropic mechanical behavior (see for example [5491410] for the face or the breast). They differ considerably from the genuine architecture of the human SSC, which is supported by a complex three dimensional collagen network causing a non-linear anisotropic mechanical behavior. Moreover, these models are dedicated to a specific anatomical area. 
Although the structure of subcutaneous tissue differs between various regions of the human body, common components have been identified in several anatomical parts. The existence of a generic pattern of organization of the SSC was put forward several years ago [11, then confirmed in recent radiological work 1] at the level of the arms, legs and trunk. Nevertheless, this anatomical finding has never been the basis of a generic modeling of the human SSC.

The aim of this work is to build, from anatomical knowledge, medical imaging and histological observation, a generic 3D geometric and mechanical model of the skin/subcutaneous complex which could be adapted to the different parts of the body and to the morphological parameters of the patient. In section 2 , we present the anatomical pattern of the SSC. In section 3, we propose a hybrid model which combines volume, membranous and unidimensional models of the different substructures. The complex internal structure of the SSC is automatically created by a procedural process. All the models are defined by some parameters which can be easily inferred by medical imaging. In section 4 , we describe several preliminary experiments which show how this method models realistic geometric deformations and physical behaviors and could be used for surgery simulation and planning.

\section{Anatomical Considerations}

\subsection{Generic Pattern of the Skin/Subcutaneous Complex}

The generic model of organization of the human skin/subcutaneous complex was confirmed by our $3 \mathrm{~T}$ MRI studies at the level of the face, the trunk and the limbs [7]. We can summarize this organization into a multi-layered model retained by a collagen network which combines the following substructures (see Fig. [1):

- Skin which has a very variable thickness (1 to $7 \mathrm{~mm}$ ) depending on locations;

- Superficial Adipose Tissue (SAT) consisting of fat lobules of variable size organized in clusters rather like palisades bands;

- Retinacula Cutis Superficialis (RCS) formed by the septal densifications lobules of the SAT;

- Membranosum Stratum (SM) or membranous layer of the superficial fascia; it can be either fibrous and/or muscular and either single or multiple;

- Deep Adipose Tissue (DAT) composed of polyhedral fat lobules, as in a honeycomb arranged more randomly than in the SAT;

- Retinacula Cutis Profondus (RCP) formed by the septal densifications lobules of the DAT;

- Skin ligaments (SL) [13], also perpendicular to the skin which are a particular specialization of RCS and RCP. They allow the maintenance of the skin or SM in deep plane or skin. They have already been described at the face, breast, the fingers, the sole or the scalp.

- Deep Layer which is the inferior limit of the SSC. This may be muscle, aponeursosis or periosteum. 


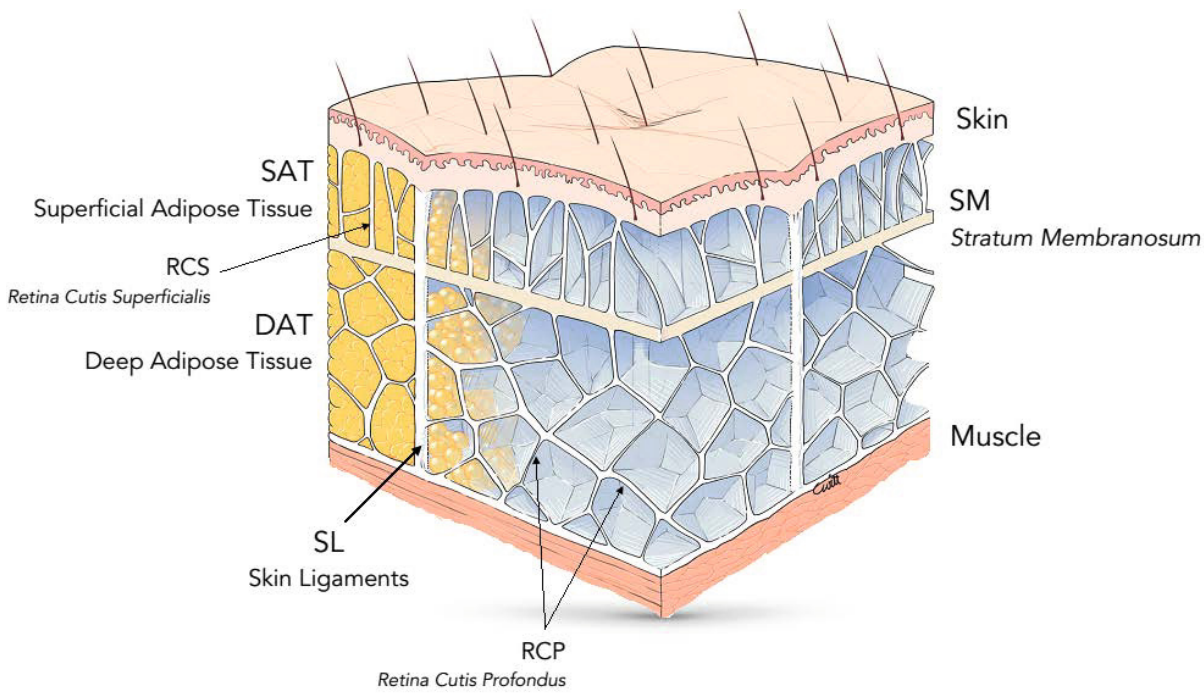

Fig. 1. Generic model of the Skin/Subcutaneous Complex with all the substructures

\subsection{Regional Specificity}

At the level of the face, the SM endorses a particular muscle specialization called Superficial Musculo Aponevrotic System (SMAS) ensuring facial expressions 9. This residue of the panniculus carnosus is found to be in continuity with the temporoparietal fascia and the superficial layer of the cervical fascia. However, this continuity is interrupted between the two nasojugal fold where the layers are intermingled, making the stratigraphic pattern more difficulty to identify.

At the level of the breast and thorax, the SM identified beyond the mammary region is continuous anteriorly, in the breast area, with a superficial layer located 3 to $4 \mathrm{~mm}$ under the skin. The collagen network, less developed in male chest, corresponds to the Cooper's ligament in female breast.

In the antero-lateral part of the abdomen, the SM forms a single layer. More anteriorly, it is divided into two parts when the adipose tissue becomes thicker. In the peri-umbilical region, the SM takes a deeper course and merges with the pre-muscular fasciae of the rectus abdominis muscles.

Finally, for the lower limbs and buttocks, the SM and Retinacula Cutis show a similar pattern of organization. A clear-cut increasing gradient in the number of $\mathrm{RC}$ in the knee and buttocks, correspond to the preferential fat storage sites.

\section{3D Modeling of the Skin/Subcutaneous Complex}

\subsection{Procedural Geometrical Modeling}

In order to deal with the variability of the SSC with respect to the regional specificity or the morphology of the patient, we introduce a procedural method 
to build a detailed 3D geometrical model. This model is based on some global parameters which can easily be measured in MRI or echography.

Skin, SM and Deep Layer are modeled by a layer of a given thickness (called respectively $\operatorname{skin}_{t}, S M_{t}$ and deep $_{t}$ ). SAT and DAT correspond respectively to the spaces between skin and SM and SM and Deep Layer, and are parameterized by their thickness $S A T_{t}$ and $D A T_{t}$. These spaces are filled by a $3 \mathrm{D}$ Voronoï tessellation where each Voronoï cell, defined by its center point, represents a fat lobule. Center points are randomly located but evenly-spaced by using the Lloyd's algorithm. The mean diameter of a fat lobule called lobule $_{d}$ gives the average distance between the center points.
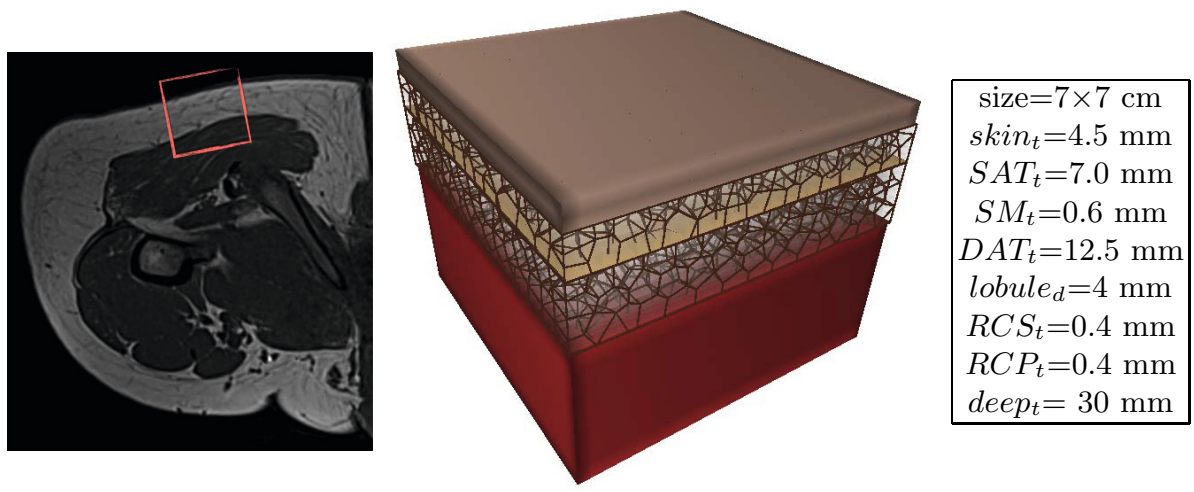

Fig. 2. SSC model for the buttocks based on parameters measured in a MR image

The walls of the Voronoï tessellation correspond to the septa of RCS and RCP which separates the lobules. They are extracted as a 3D triangle mesh. We assume plane stress conditions for these walls, meaning that we neglect bending over stretching; so wall thickness is not geometrically represented.
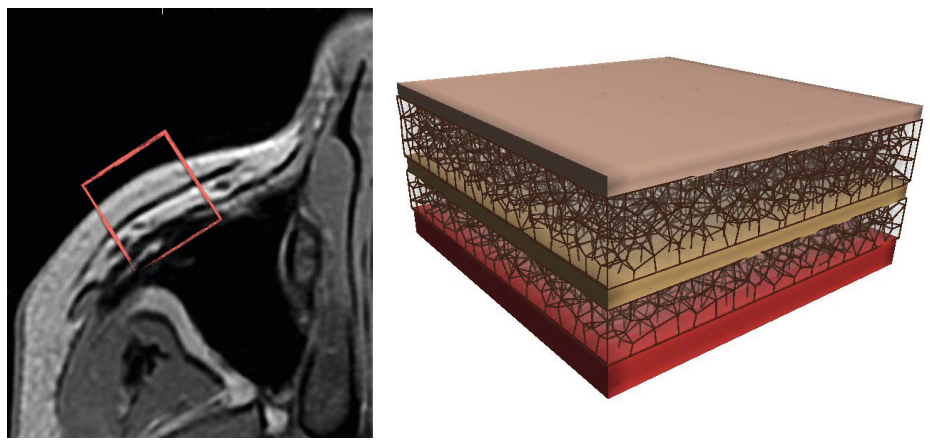

size $=3 \times 3 \mathrm{~cm}$
skin $_{t}=1.2 \mathrm{~mm}$
$S A T_{t}=5.0 \mathrm{~mm}$
$S M_{t}=1.2 \mathrm{~mm}$
DAT $_{t}=3.4 \mathrm{~mm}$
lobule $_{d}=1.5 \mathrm{~mm}$
$R C S_{t}=0.2 \mathrm{~mm}$
$R C P_{t}=0.1 \mathrm{~mm}$
deep $_{t}=2.5 \mathrm{~mm}$

Fig. 3. SSC model for the cheek based on parameters measured in a MR image 
Fig. 2 and 3 demonstrate that specificity of the anatomical region is taken into account. At the level of the buttocks, the skin is thick, the thickness of the DAT is very variable depending on the location. The mesh formed by the RCS and RCP is very dense in areas of fat storage. At the level of the face, the SM, thicker, has a muscle differentiation. The volume of lobules is small and RCS as $\mathrm{RCP}$ are thin.

\subsection{Hybrid Mechanical Modeling}

From a mechanical point of view, the SSC is composed of elements of variable mechanical behaviors: hyperelastic for the skin and visco-elastic for the fat. This assembly has a global non-linear anisotropic behavior principally due to the existence and orientation of collagen fibers. Detailing the stratigraphic and lobular organization of the sub-cutaneous fat, we wanted to recreate the overall anisotropy of the SSC by adding linear isotropic elements. This approximation is permitted by the architectural complexity of the geometrical model.

To model the different substructures, we built an hybrid model. It combines a volume model for the skin, SM, SAT and DAT, a membranous model for the interlobular septa of RCS and RCP and a unidimensional model for the skin ligaments.

We used the SOFA framework [3] which is particularly suitable for implementing and processing this type of composite models in nearly real-time. Programming is based on a Python script that allows flexibility in the geometrical and mechanical parameterization.

Volume, membranous and unidimensional components of our model were discretized using respectively hexahedral, triangular and edge finite elements. To mechanically couple them, these elements were embedded in a coarser regular grid using barycentric interpolation. The typical size of a coarse hexahedron was $2 \mathrm{~mm}$. Time integration for coarse nodes was performed using the Euler implicit scheme and conjugate gradient algorithm. Strain was measured using the corotational method.

For each substructure, we extracted Young's modulus data from the literature, and performed an average. We try to assess these values by a simulation of indentation in similar conditions of some published research. Poissons ratio was fixed at 0.49 due to the incompressible nature of the materials.

\section{Some Experiments}

\subsection{Simulation of Indentation}

In order to validate the behavior of our model, we simulated an indentation test of the forearm with the parameters found in an in vivo MRI study [15]. Geometrical parameters are listed in Fig. 4, right. In paticular, DAT is very thin. Young modulus of skin, fat, SM, RC and muscle were respectively set to 20, $1,60,100$ and $15 \mathrm{kPa}$. 
The indenter was modeled as a cylinder of diameter $5 \mathrm{~mm}$. Such as in the experiment presented in [15], we simulated several indentations between 0.35 and 1.8 N. Fig. 4, shows a comparison between real data and the obtained vertical displacements at equilibrium. On average the error was $18 \%$ suggesting that our model is rather realistic.

Accuracy could be improved in future using non-linear material laws. However, this will raise the problem of parameter identification, as a high discrepancy in the reported material parameter can be found in the literature, depending on the measurement modality and test specimen.
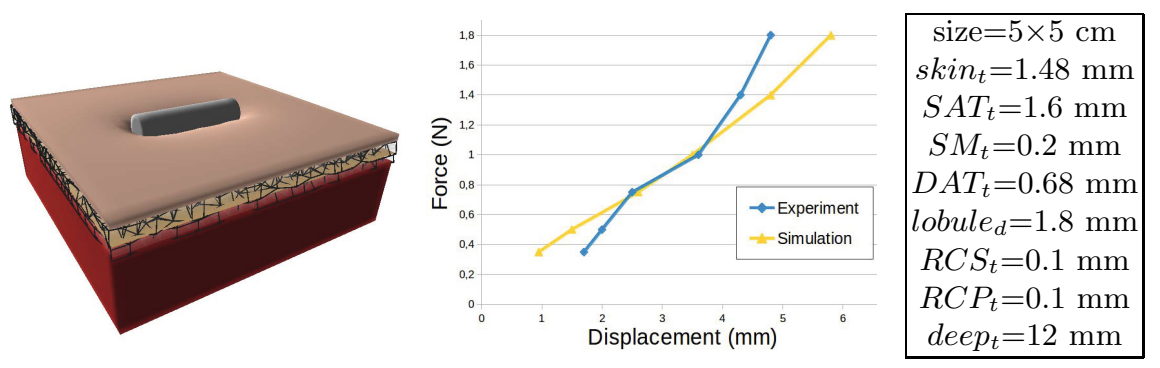

Fig. 4. Simulation of indentation of the forearm according to an in vivo MRI study

\subsection{Simulation of the Phenomenon of Cellulite}

Cellulite is considered for the majority of authors as a consequence of the increased content of fat lobes associated with a deterioration of collagen tissue of the skin and the relative stability of RC underlying the skin [8. We simulated the increase of $24 \mathrm{~cm}^{3}$ of fat in the SAT and DAT of the buttocks model of Figure 2 by modifying the rest shape of fat material. This results in skin dimpling and nodularity as shown in Fig. 5 left. Then we reduced the stiffness of the skin to simulate aging. Cellulite is enhanced as we can see in Fig. 5 right.

\subsection{Simulation of Fat Injection in the Subcutaneous Tissue}

Fat grafting is a major technique in reconstructive surgery. During this procedure, the injection site significantly affects the outcome. In a deep injection (that is in DAT), the effect is said "volumizer" and the main purpose is usually to increase the projection of an anatomical structure (cheekbone, buttocks, breasts...). In contrast, if the injection is more superficial, the effect is more on the shape of the injection site and it is most often used to fill wrinkles or scars.

We simulated an injection of $0.5 \mathrm{~cm}^{3}$ of fat inside the DAT of the cheek model of Fig. 3. It results in a smooth and global elevation of the skin surface (see Fig. 6 left). Fig. 6 right shows the simulation of a similar injection inside the SAT. Our model allows to simulate the "orange peel" phenomenon with a high fidelity. This problem during surface injection is common in daily clinical practice and feared if a too large volume of injection is carried out in a too superficial plane. 

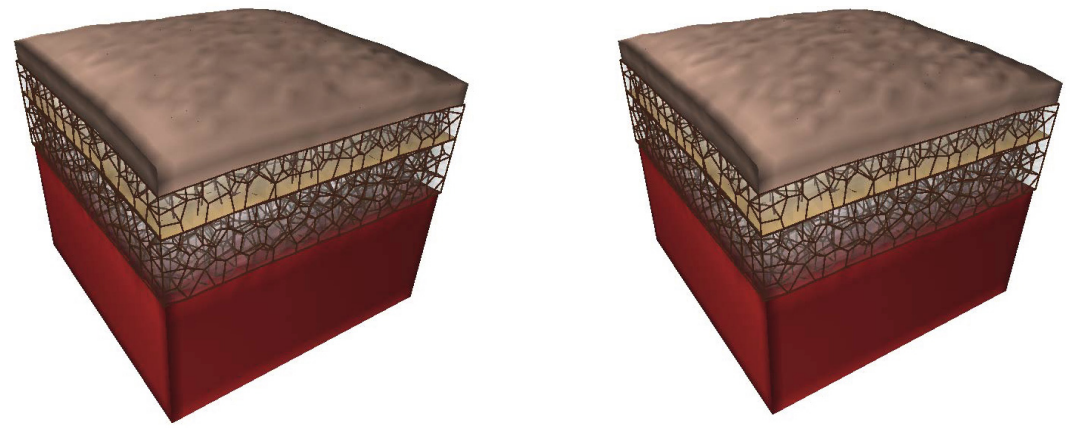

Fig. 5. Simulating cellulite in buttocks by increasing fat volume in SAT and DAT. Right: cellulite enhancement due to aging simulation.
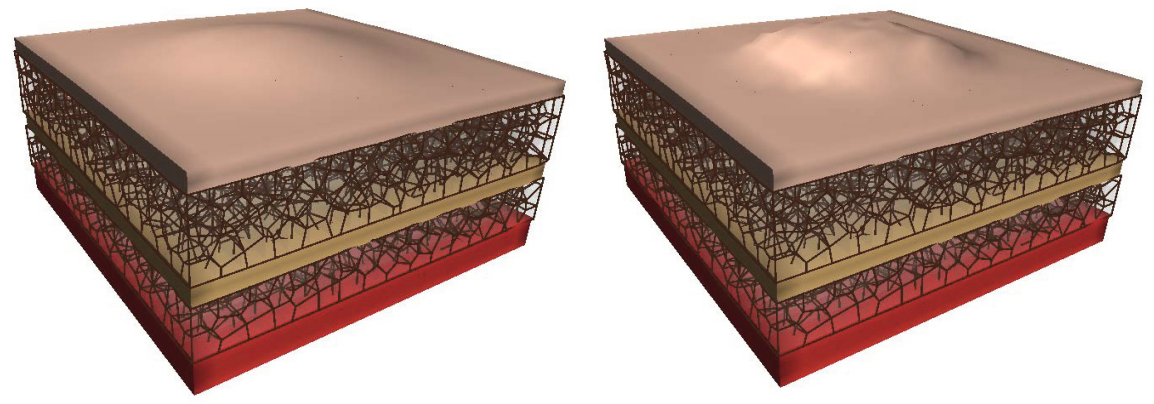

Fig. 6. Simulating fat injection in the cheek at the level of DAT (left) and SAT (right). If the injection is too superficial, the "peel orange" phenomenon appears.

\section{Future Work}

If we have the external shape of the patient, for example by using MRI or a $3 \mathrm{D}$ surface scanner, we could deform the generic model and fit it to his specific and exact morphology. Such a registration will allow us to perform pre-operative simulations of plastic surgeries of the face, the thorax or the breast. In particular, we think that our model will be able to simulate breast augmentation surgery in a more faithful way because it includes an accurate modeling of RC, which corresponds to the Cooper's ligament which has a major role in the final shape of the breast.

This model will also permit to simulate fat grafting inside the adipose tissue taking into account its lobular composition and its retaining connective tissue. Some studies focus on fat injection as [6] and more recently [12] but none is able to simulate fat grafting considering the destruction of septa or skin ligaments. Such a technique called fasciotomy is common in daily practice, in a particular in breast reconstruction [2. The simulation could be done procedurally by deleting the walls of the Voronoî cells corresponding to the RCS and cutting the skin ligaments. 
Nevertheless, to simulate a more accurate behavior of the skin, it will be necessary to add to our model the anisotropic behavior of the skin and the preexisting tension. These properties have been described in details and are often represented as Langer's lines. This would allow us to simulate other type of plastic surgeries as skin incision or lifting.

Acknowledgments. We want to thank the radiology team of University Hospital of Nîmes, France for MR acquisitions and Ms. Witt for the quality of her drawings.

\section{References}

1. Abu-Hijleh, M.F., Roshier, A.L., Al-Shboul, Q., Dharap, A.S., Harris, P.F.: The membranous layer of superficial fascia: evidence for its widespread distribution in the body. Surgical and Radiologic Anatomy 28(6), 606-619 (2006)

2. Agha, R., Fowler, A., Herlin, C., Goodacre, T., Orgill, D.: Use of autologous fat grafting for reconstruction post-mastectomy and breast conserving surgery: A systematic review and meta-analysis. European Journal of Surgical Oncology 40(5), 614-615 (2014)

3. Allard, J., Cotin, S., Faure, F., Bensoussan, P.J., Poyer, F., Duriez, C., Delingette, H., Grisoni, L.: SOFA - an open source framework for medical simulation. In: MMVR 15 - Medicine Meets Virtual Reality, vol. 125, pp. 13-18 (February 2007), http://hal.inria.fr/inria-00319416

4. Barbarino, G., Jabareen, M., Trzewik, J., Mazza, E.: Physically based finite element model of the face. In: Bello, F., Edwards, E. (eds.) ISBMS 2008. LNCS, vol. 5104, pp. 1-10. Springer, Heidelberg (2008)

5. Chabanas, M., Luboz, V., Payan, Y.: Patient specific finite element model of the face soft tissues for computer-assisted maxillofacial surgery. Medical Image Analysis $7(2), 131-151(2003)$

6. Comley, K., Fleck, N.: Deep penetration and liquid injection into adipose tissue. Journal of Mechanics of Materials and Structures 6(1-4), 127-140 (2011)

7. Herlin, C., Chica-Rosa, A., Subsol, G., Gilles, B., Macri, F., Beregi, J., Captier, G.: Three-dimensional study of the skin/subcutaneous complex using in vivo whole body 3 tesla MRI. review of the literature and confirmation of a generic pattern of organisation. Accepted for Publication in Surgical and Radiologic Anatomy (2014)

8. Hexsel, D., Siega, C., Schilling-Souza, J., Porto, M.D., Rodrigues, T.C.: A comparative study of the anatomy of adipose tissue in areas with and without raised lesions of cellulite using magnetic resonance imaging. Dermatologic Surgery 39(12), 1877-1886 (2013)

9. Hung, A.P.L., Wu, T., Hunter, P., Mithraratne, K.: A framework for generating anatomically detailed subject-specific human facial models for biomechanical simulations. The Visual Computer, 1-13 (May 2014)

10. Lapuebla-Ferri, A., del Palomar, A.P., Herrero, J., Jiménez-Mochol, A.J.: A patientspecific FE-based methodology to simulate prosthesis insertion during an augmentation mammoplasty. Medical Engineering \& Physics 33(9), 1094-1102 (2011)

11. Lockwood, T.E.: Superficial fascial system (SFS) of the trunk and extremities: a new concept. Plastic and Reconstructive Surgery 87(6), 1009-1018 (1991) 
12. Majorczyk, V., Cotin, S., Duriez, C., Allard, J.: Simulation of lipofilling reconstructive surgery using coupled eulerian fluid and deformable solid models. In: Mori, K., Sakuma, I., Sato, Y., Barillot, C., Navab, N. (eds.) MICCAI 2013, Part III. LNCS, vol. 8151, pp. 299-306. Springer, Heidelberg (2013)

13. Nash, L.G., Phillips, M.N., Nicholson, H., Barnett, R., Zhang, M.: Skin ligaments: regional distribution and variation in morphology. Clinical Anatomy 17(4), 287-293 (2004)

14. Roose, L., De Maerteleire, W., Mollemans, W., Maes, F., Suetens, P.: Simulation of soft-tissue deformations for breast augmentation planning. In: Harders, M., Székely, G. (eds.) ISBMS 2006. LNCS, vol. 4072, pp. 197-205. Springer, Heidelberg (2006)

15. Tran, H.V.: Caractérisation des propriétés mécaniques de la peau humaine in vivo via l'IRM. Ph.D. thesis, Université de Technologie de Compiègne (October 2007) 